\title{
Zur Bedeutung von Normativität in den Bildungswissenschaften
}

\section{Caroline Villiger Hugo, Nicolas Perrin, Sandrine Aeby Daghé}

Das französischsprachige und das deutschsprachige Editorial gehen in der vorliegenden Ausgabe der Schweizerischen Zeitschrift für Bildungswissenschaften getrennte Wege. Dies ergibt sich aus dem einfachen Grund, weil die beiden Sprachkulturen auf eine unterschiedliche historische Entwicklung der Disziplin zurückblicken, was die Herangehensweise an das Thema "Normativität in den Bildungswissenschaften» entsprechend beeinflusst. So bietet dieses Editorial für die gemeinsame Ausgangsproblematik bewusst zwei unterschiedliche, sprachkulturabhängige Einführungen in das gewählte Varia-Thema an. Wir laden die deutschsprachigen Leserinnen und Leser deshalb ein, sich auf diese sprachkulturelle Auseinandersetzung einzulassen und das französischsprachige Editorial ebenfalls zu beachten.

Eine Literaturrecherche im deutschsprachigen Raum zeigt, dass die Diskussion über Normativität in den Bildungswissenschaften lediglich einen Nebenschauplatz besetzt (Fuchs, May \& Krause, 2013). Warum ist Normativität derzeit kein Thema? Gehört Normativität einer veralteten Vorstellung von Erziehungswissenschaft an und ist deshalb aus der aktuellen Debatte innerhalb der Bildungswissenschaften kommentarlos verdrängt worden? Eine historische Betrachtung der Entwicklung unserer Disziplin kann bei der Klärung dieser Fragen hilfreich sein.

Ein neuralgischer Wendepunkt ergab sich Mitte des 20. Jahrhunderts, als die ursprünglich normativ orientierte, geisteswissenschaftliche Pädagogik bei der Bearbeitung gewisser Fragestellungen mit den Grenzen der bislang üblichen Methoden konfrontiert war. So konnten schulpraktische Herausforderungen wie beispielsweise die Frage nach der Effizienz von Bildungseinrichtungen oder nach der gerechten Verteilung von Bildungschancen mit den traditionellen philosophisch-geisteswissenschaftlichen Methoden nicht auf befriedigende Weise beantwortet werden (Reble, 1995). Die Forderung des deutschen Pädagogen und Erziehungswissenschaftlers Heinrich Roth bestand darin, zusätzlich zu der normativen Ausrichtung, die für die Pädagogik unabdingbar sei, auch erfah- 
rungswissenschaftliche Forschungsmethoden in der Pädagogik einzusetzen (Roth, 1962). Damit hat er im deutschsprachigen Raum eine neue methodologische Epoche in der Erziehungswissenschaft eingeleitet, die, inspiriert von den damals bereits angewendeten sozialwissenschaftlichen Methoden in den USA, in der empirischen Bildungsforschung bis heute andauert. Auch andere Erziehungswissenschaftler haben diesen Standpunkt vehement verteidigt und sind zum Teil in ihren Forderungen noch weiter gegangen: Brezinka (1972) forderte eine wertfreie Erziehungswissenschaft, die den Praktikerinnen und Praktikern keine Vorschriften macht. Die Wissenschaftlichkeit der Disziplin bestehe darin, dass durch die Wissenschaft generierte Aussagen intersubjektiv überprüfbar und rein deskriptiv, nicht aber präskriptiv seien. Brezinka gehörte damit einer Denkweise an, die Pädagogik als kritisch-rationale empirische Wissenschaft (eben: Erziehungswissenschaft) in der Tradition des kritischen Rationalismus von Karl Popper (1973) sieht.

Der heutige Stand bzw. das Ergebnis dieser Entwicklung ist ein Nebeneinander von beiden Ansichten oder besser noch: ein Spektrum von Ansichten innerhalb der heutigen Erziehungs- und Bildungswissenschaften, die sich zwischen einer rein geisteswissenschaftlichen, normativen und einer im Extremfall ganz der Normativität abgewandten, kritisch-analytischen Orientierung bewegen. Dies ist objektiv gesehen als ein Reichtum der Disziplin zu verzeichnen, obschon dies von einzelnen Vertreterinnen und Vertretern der erziehungswissenschaftlichen Forschung längst nicht immer als solcher wahrgenommen wird. Gleichzeitig kann mit Terhart (2006) kritisch festgehalten werden, dass das Verhältnis von normativ-geisteswissenschaftlicher und empirisch-analytischer Erziehungswissenschaft zu sehr einem Antagonismus verhaftet ist. Beide "Communities» würden aber zu wenig anerkennen, dass sie sich gegenseitig bedingen, bzw. dass das eine im anderen dennoch enthalten ist (vgl. dazu Oelkers, 2014). Die empirische Bildungsforschung führe «bestimmte, weitgehend unexpliziert bleibende, stille bildungsphilosophische oder doch bildungsphilosophisch relevante Argumentationen und Positionen mit sich, die im Interesse Aller expliziert gehören.» (Terhart, 2006, S. 9). Umgekehrt nutze die Bildungsphilosophie stillschweigend Annahmen und Argumente, die im Grunde genommen empirischer Natur seien, jedoch als solche nicht expliziert und geprüft werden. Dieses unausweichliche Verhältnis anzuerkennen und besser als bisher zu klären, d.h. ihre jeweiligen empirischen oder normativen Bezüge expliziter zu machen darin liegt eine Entwicklungsmöglichkeit für beide «Communities», so Terhart.

Eines ist klar: Bildungsforschung - auch empirische - kann sich der Normativität nicht gänzlich entziehen. Auch Bildungsverantwortliche, oftmals die Adressaten von Bildungsforschern, orientieren sich an Angemessenheit, Güte, Normen oder zumindest "Wünschbarem» in Bezug auf erzieherisches Handeln oder auch bei der Gestaltung von Bildungsprozessen und Bildungspolitik. Wenn wir die Landschaft der heutigen Bildungswissenschaften genauer betrachten, dann sind an vielen Stellen mehr oder weniger explizite Bezüge zu Normen oder Werten 
erkennbar. Die meisten der in dieser Varia-Nummer publizierten Beiträge zeigen ebenfalls, dass bildungswissenschaftliche Fragestellungen nicht losgelöst von normativen Bezügen bearbeitet werden können. Aufgrund der eingegangenen Artikel haben wir einen Versuch der Klassifizierung unternommen und dabei folgende Normativitätsbezüge in der erziehungswissenschaftlichen Forschung ausmachen können: A. Orientierung an sozial anerkannten Werten und Normen und gegebenenfalls Überprüfung ihrer Berücksichtigung in der Praxis, B. Vorgabe von theoriegeleiteten bzw. evidenz-basierten Standards, Rahmenkonzepten oder Qualitätsmerkmalen, die für die Praxis handlungsleitenden Charakter haben, und schliesslich C. Überprüfung des Vorhandenseins wissenschaftlich anerkannter Normen, Standards oder Qualitätsmerkmale in der Praxis bzw. in der berufspraktischen Ausbildung. Selbstverständlich handelt es sich bei dieser Klassifizierung um ein Raster, das keine Allgemeingültigkeit beansprucht, sondern induktiv aus den vorliegenden Artikeln hergeleitet worden ist. In der Folge werden die Artikel in Anlehnung an diese Klassifizierung kurz skizziert und eingeordnet.

\section{Orientierung an sozial anerkannten Werten und Normen (und gegebenenfalls überprüfung ihrer Berücksichtigung in der Praxis)}

Mit Cyberbullying ist eine Thematik gegeben, die sich auf den ethisch-moralisch unkorrekten Umgang mit Anderen via elektronischer Kommunikationsmittel bezieht. Im Bewusstsein um die gesundheitlichen Auswirkungen von derartigem Verhalten auf Opfer befassen sich Müller, Hofmann, Hinni, Müller, Begert und Zurbriggen in ihrer Studie mit der berichteten Häufigkeit und Formen von Cyberviktimisierung bei Schülerinnen und Schülern der 7. Klasse sowie mit Bildungsgangunterschieden. Weiter untersuchen die Autoren, ob die höhere Ausprägung der kommunikativen Nutzung elektronischer Medien die Bildungsgangunterschiede zu erklären vermag. Schultypabhängige Entwicklungsmilieus und damit verbundene Risiken ungünstiger Klassennormen sowie der explizite Aufbau prosozialen Verhaltens als präventive Massnahme gegen Cyberbullying werden diskutiert.

Die Arbeit von Villemonteix behandelt die herkömmlichen Praktiken und Äusserungen von Primarlehrpersonen über ihre Nutzung von Informationstechnologien wie beispielsweise die interaktive Wandtafel und der Tablet-Computer. Ziel ist zu analysieren, ob diese neueren didaktischen Mittel den «klassischen» Unterricht verändern. Dabei stehen die Entstehungsgeschichte von technologischen Apparaten, die Veränderung der Schüleraufträge sowie die Funktion der Arktefakte im Fokus der Analysen. Er zeigt, dass «die Kunst des Lehrmeisters» oftmals fortbesteht und die neuen Technologien das klassische Schema von Übermittlung/Einübung/Einzelevaluation in keiner Weise bricht. Verbunden mit der Frage der Normativität sind insbesondere die Komplexität der Handhabung derartiger Technologien und die Möglichkeit, Schule zu 
verändern.

Zwingenberger und Obrecht untersuchen in ihrem Beitrag den Zusammenhang von den kantonal variierenden gymnasialen Maturitätsquoten und den Studienleistungen der Studierenden im Eintrittsjahr an der Universität St. Gallen. Dahinterliegend lässt sich die Frage nach der angemessenen Höhe der Schweizer Maturitätsquote erkennen, mit dem normativen Ziel einer besseren Ausschöpfung der Bildungsressourcen sowie einer Verringerung sozialer Selektionseffekte. Im weiteren Sinn geht es also um Bildungsgerechtigkeit sowie um die Frage, ob eine Erhöhung der gymnasialen Maturitätsquote in Kantonen mit geringer Quote Herkunftseffekte minimieren könnte.

Soussi untersucht das Programm REP (réseau d'enseignement prioritaire) in Genf im Vergleich du ähnlichen Bildungspolitiken in Frankreich, Belgien und in Zürich. Dabei hebt sie als einen gemeinsamen Kernpunkt die Irritation öffentlicher Institutionen und Akteure angesichts der Problematik des schulischen Misserfolg und den Ungleichheiten hervor. Die untersuchten Bedingungen (Festlegung der Population, Art der angewendeten Aktionspläne, frühzeitige Interventionen) führen die Autorin dazu, sich zu fragen, ob ein kompensatorischer Ansatz genügt oder ob ein transformatorischer Ansatz besser wäre (und wenn ja, welcher). Sie schliesst mit der Erkenntnis, dass man sich fragen muss, ob die Ziele in den meisten Fällen wirklich erreicht sind oder ob nicht vielmehr das Schlimmere verhindert werden konnte angesichts der sozialen Verschlechterung in gewissen Vierteln.

Vorgabe von theoriegeleiteten oder evidenzbasierten Standards/Rahmenkonzepten/Qualitätsmerkmalen, die für die Praxis handlungsleitenden Charakter haben

Bei dem Beitrag von Reinbacher handelt es sich um den Versuch, dem Feld «Schulentwicklung» in der Tradition von Talcott Parsons und Helmut Fend einen integrativen, theoriegeleiteten Orientierungsrahmen zu geben. Mit der Einbettung schulischer Entwicklungsprozesse in gesellschaftliche Strukturzusammenhänge soll dieses Konzept ein Bezugsrahmen für Detailanalysen zu einzelnen Dimensionen von Schulentwicklung sowie zur Ortung von potentiellen Schulentwicklungsvorhaben sein, aber auch zum Verstehen von Hintergründen verschiedener Zugänge zu Systemsteuerung und Systemreflexion beitragen. Der Artikel beabsichtigt, mittels seiner theoriegeleiteten Vorgehensweise den «normativen Fallstricken» im Gebiet der Schulentwicklung dezidiert entgegenzuwirken. Gleichzeitig wird an mehreren Stellen deutlich, welche Bedeutung Normen und Werten innerhalb des Systems Schule zukommt, und dass deren Berücksichtigung bei der Schulentwicklung eminent wichtig ist.

Die Studie von Alincai und Weil-Barais untersucht ein Programm zur Elternbildung. Im Anschluss an einen Literaturüberblick präsentiert der Beitrag das Konzept und die Umsetzung eines Elternbildungsprogramms im Rahmen eines Museumsbesuchs. Dieses Programm beinhaltet die Videoaufzeichnung von 
Interaktionen zwischen Eltern und Kindern und von Gesprächen zwischen einem Animator und den Eltern. Die Autoren schlussfolgern, dass die Frage der Normativität der Programme oft im Zentrum der Kritik steht, als ob die Normativität bei jeglicher Bildung unbedingt abwesend sein sollte. Vielmehr schlagen sie vor, den Anteil an Normativität, den jeder Erzieher zu erfüllen bereit sein sollte, zu definieren. In der Tat gäbe es keine Erziehung ohne Teilhabe an denselben Normen.

Überprüfung des Vorhandenseins wissenschaftlich anerkannter Normen/Standards/Qualitätsmerkmale in der Praxis bzw. berufspraktischen Ausbildung

Wanlin präsentiert eine Untersuchung zur Unterrichtsplanung und zeigt die Funktionen der Planung und die von den Lehrpersonen benutzten Denkmodelle auf. Er kontrastiert dabei zwei Ausrichtungen (Zielorientierung/Inhaltsorientierung oder den Schülern aufgetragene Aktivitäten). Dabei macht er deutlich, dass mehrere in der Literatur erwähnte Gegenüberstellungen für die befragte Stichprobe nicht brauchbar sind. Interessanterweise weist der Autor sogleich darauf hin, dass die Unterrichtsplanung ein entscheidender Faktor für die Führung eines guten Unterrichts und ein effizientes Lernen sei. Ausserdem beendet er den Artikel mit einem Abschnitt, in dem er die Folgerungen für die Grundausbildung von Lehrpersonen skizziert. Die Frage des Wünschbaren stellt sich also zu Beginn und zum Schluss der Forschungsarbeit.

Marcoux, Boraita und Crahay untersuchen den Zusammenhang zwischen den Einstellungen der berufstätigen und den angehenden Lehrpersonen bezüglich der Klassenwiederholung und ihre Kenntnisse von Forschungsergebnissen über die Wirkung von Klassenwiederholung. Ihre Studie zeigt die Auswirkungen des ersten Ausbildungsjahres auf die Einstellungen. Gleichzeitig erklärt sie die Hypothese, dass sich diese Einstellungen auf gefestigte Konzepte gründen, für ungültig. Eine Erklärung dafür könnte in der Tatsache liegen, dass die befragten Personen dem sozialen Druck in der Praxis, wo man an der Wirksamkeit der Klassenwiederholung festhält, wenig Widerstand leisten. Aus dieser Sicht ist die Praxis der Klassenwiederholung nicht nur eine Folge von Einstellungen, und diese zu verändern wäre daher ungenügend: Änderungen auf institutioneller Ebene seien ebenso erforderlich.

Fregonese und Ratinaud untersuchen in ihrem Beitrag wie angehende Hebammen im Rahmen ihrer fünfjährigen Ausbildung den eigenen Beruf und den der Ärztin oder des Arztes sehen. Die Ergebnisse dieser Studie bieten Anlass, das «Engineering» der Ausbildung in Frage zu stellen und über Anpassungen nachzudenken. Auch können Kenntnisse über diese kognitiven Repräsentationen bei der Auslotung von Ausbildungszielen und Erwartungen von Seiten der Berufspraxis von entscheidender Bedeutung sein. 


\section{Literatur}

Brezinka, W. (1972). Von der Pädagogik zur Erziehungswissenschaft, 2. Auflage. Weinheim: Beltz.

Fuchs, T., May, J. \& Krause, S. (2013, Hrsg.). Normativität und Normative (in) der Pädagogik. Würzburg: Königshausen \& Neumann.

Oelkers, J. (2014). Empirie oder Philosophie? Ein internationales Spannungsfeld der Pädagogik. Pädagogische Rundschau, 68, 445-462.

Popper, K. (1973). Objektive Erkenntnis. Ein evolutionärer Entwurf. Hamburg: Hoffmann und Campe.

Reble, A. (1995). Geschichte der Pädagogik, 18. Aufl. Stuttgart: Klett-Cotta.

Roth, H. (1962). Die realistische Wendung in der Pädagogischen Forschung. In H. Becker, E. Blochmann, O. F. Bollnow, E. Heimpel \& M. Wagenschein (Hrsg.). Neue Sammlung. Göttinger Blätter für Kultur und Erziehung. 2. Jg. Göttingen: Vandenhoeck \& Ruprecht.

Terhart, E. (2006). Bildungsphilosophie und empirische Bildungsforschung - (k)ein Missverhältnis? In L. Pongratz, M. Wimmer \& W. Nieke (Hrsg.). Bildungsphilosophie und Bildungsforschung (S. 9 -36). Bielefeld: Janus Presse. 